\title{
Endovascular Treatment of Middle Cerebral Artery Aneurysms: Are We There Yet?
}

\author{
Wim H. van Zwam $^{1}$ (]
}

Received: 29 December 2020/ Accepted: 20 January 2021/Published online: 1 February 2021

(C) Springer Science+Business Media, LLC, part of Springer Nature and the Cardiovascular and Interventional Radiological Society of Europe (CIRSE) 2021

Since publication of ISAT [1], endovascular coiling has become the first treatment choice in most centers and for most intracranial aneurysms. However, basilar artery and middle cerebral artery (MCA) aneurysms were underrepresented in this trial because of assumed lack of equipoise by participating physicians. Basilar artery aneurysms because of difficult accessibility for open surgery while in most cases relatively easily treatable by endovascular coiling. For MCA aneurysms, these arguments are generally reversed: relatively easily accessible for open surgery and often difficult to treat with coils due to complex anatomy with wide neck and/or branches originating from the neck of the aneurysm. While the verdict for basilar aneurysms has been quite uniform 'coiling first', the debate about MCA aneurysms is still ongoing. In general, clipping has appeared to have slightly worse clinical outcome [1], while coiling more often leads to incomplete occlusion or recanalization over time, requiring imaging follow-up and retreatments in up to $10 \%$ of cases [2].

A systematic review on clipping and coiling of MCA aneurysms appeared in 2016 including 25 studies with 1149 coiled and 15 studies with 742 clipped ruptured MCA aneurysms [3]. Despite the lack of standardization in reported data and the assumed bias in published studies, they concluded that both clipping and coiling have low mortality and morbidity rates and that coiling seems preferable for ruptured MCA aneurysms (and clipping for unruptured MCA aneurysms) but that no firm conclusions

Wim H. van Zwam

w.van.zwam@mumc.nl

1 Maastricht University Medical Centre, 6202 AZ Maastricht, The Netherlands can be drawn and standardized observational studies from prospectively kept databases are needed to allow for stronger conclusions to be made.

The current publication by Hagen et al. [4] could contribute to this need for more data. They present clinical and angiographic results of 118 endovascularly treated ruptured MCA aneurysms, not all with coils, as also the more recent 'endovascular flow disruption' technique with the WEB device (Woven EndoBridge; Sequent Medical, Aliso Viejo, California) is included. Clinical outcomes (mRS) at discharge, occlusion grade (Raymond Roy Occlusion Classification [RROC]) and periprocedural complications are reported as well as follow-up data including clinical outcome, retreatment rate and final occlusion grade.

Periprocedural complication rate, complete occlusion and retreatment rate are more or less conform previous studies. Still, a retreatment rate of up to $9.5 \%$ (for WEBtreated aneurysms) should be a point of concern as the alternative, surgical clipping, goes with considerably lower retreatment rates. This higher rate of retreatments was also reported in their series of WEB-treated unruptured MCA aneurysms [5]. However, they attributed this to a learning curve for use of the WEB device.

In many centers where a 'clipping first' strategy for MCA aneurysms is followed, only aneurysms with a favorable geometry, or patients with contraindications for open surgery, are treated endovascularly. In the current unselected cohort, a subgroup analysis on aneurysms with an unfavorable geometry (small dome-to-neck ratio, branches from the base of the aneurysm) could have given direction towards a better-founded choice between coiling and clipping. Unfortunately, this has not been done. Therefore, although the overall outcome may justify the conclusion that endovascular treatment of ruptured MCA 
aneurysms is feasible and safe, it will not convince followers of the clipping first adagium to change their strategy. Moreover, the WEB device does not appear to be the solution for MCA aneurysms with unfavorable geometry in this study cohort. The choice between coiling and clipping of MCA aneurysms will remain dependent on patient and aneurysm characteristics, as well as on local preferences and experience.

\section{Funding None.}

\section{Compliance with ethical standards}

Conflict of interest Speaker fees from Stryker and Cerenovus, both paid to Institution.

Consent for publication For this type of study, consent for publication is not required.

\section{References}

1. Molyneux A, et al. International Subarachnoid Aneurysm Trial (ISAT) of neurosurgical clipping versus endovascular coiling in
2143 patients with ruptured intracranial aneurysms: a randomised trial. Lancet. 2002;360(9342):1267-74.

2. Ferns SP, et al. Coiling of intracranial aneurysms: a systematic review on initial occlusion and reopening and retreatment rates. Stroke. 2009;40(8):e523-9.

3. Zijlstra IA, et al. Coiling and clipping of middle cerebral artery aneurysms: a systematic review on clinical and imaging outcome. J Neurointerv Surg. 2016;8(1):24-9.

4. Hagen F, Berlis A, Skalej M, et al. Endovascular treatment of ruptured middle cerebral artery bifurcation aneurysms. a retrospective observational study of short- and long-term follow-up. Cardiovasc Intervent Radiol. 2020. https://doi.org/10.1007/ s00270-020-02718-9.

5. Hagen F, Maurer CJ, Berlis A. Endovascular treatment of unruptured MCA bifurcation aneurysms regardless of aneurysm morphology: short- and long-term follow-Up. AJNR Am J Neuroradiol. 2019;40(3):503-9.

Publisher's Note Springer Nature remains neutral with regard to jurisdictional claims in published maps and institutional affiliations. 